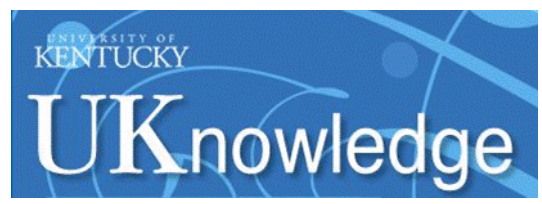

University of Kentucky

UKnowledge

\title{
Response Style Differences in the Inattentive and Combined Subtypes of Attention-Deficit/Hyperactivity Disorder
}

\author{
Karen J. Derefinko \\ University of Kentucky, karen.derefinko@uky.edu \\ Zachary W. Adams \\ University of Kentucky, zach.adams@uky.edu \\ Richard Milich \\ University of Kentucky, richard.milich@uky.edu \\ Mark T. Fillmore \\ University of Kentucky, fillmore@uky.edu \\ Elizabeth P. Lorch \\ University of Kentucky, elorch@uky.edu
}

See next page for additional authors

Follow this and additional works at: https://uknowledge.uky.edu/psychology_facpub

Part of the Child Psychology Commons

Right click to open a feedback form in a new tab to let us know how this document benefits you.

\section{Repository Citation}

Derefinko, Karen J.; Adams, Zachary W.; Milich, Richard; Fillmore, Mark T.; Lorch, Elizabeth P.; and Lynam, Donald R., "Response Style Differences in the Inattentive and Combined Subtypes of Attention-Deficit/ Hyperactivity Disorder" (2008). Psychology Faculty Publications. 1.

https://uknowledge.uky.edu/psychology_facpub/1

This Article is brought to you for free and open access by the Psychology at UKnowledge. It has been accepted for inclusion in Psychology Faculty Publications by an authorized administrator of UKnowledge. For more information, please contact UKnowledge@lsv.uky.edu. 


\section{Response Style Differences in the Inattentive and Combined Subtypes of Attention-Deficit/Hyperactivity Disorder}

Digital Object Identifier (DOI)

$10.1007 /$ s10802-007-9207-3

\section{Authors}

Karen J. Derefinko, Zachary W. Adams, Richard Milich, Mark T. Fillmore, Elizabeth P. Lorch, and Donald R. Lynam 
Running head: ADHD SUBTYPES

Response Style Differences in the Inattentive and Combined Subtypes of AttentionDeficit/Hyperactivity Disorder 


\begin{abstract}
This study examined potential differences between the inattentive and combined ADHD subtypes using laboratory tasks assessing behavioral inhibitory processes. Seventy-five children completed two tasks of behavioral inhibition believed to isolate different processes: the cued reaction time task (CRT), a basic inhibition task, and the go/no-go task (GNG), a complex inhibition task that incorporates motivational contingencies. Three groups of participants were identified, including ADHD/Inattentive $(n=17)$, ADHD/Combined $(n=37)$, and comparison $(n$ =21). Results indicated that rather than showing behavioral inhibition deficits, the ADHD/I children appeared overly inhibited, as evidenced by slower reaction times across the two tasks and significantly higher errors of omission in the GNG task. Additionally, the ADHD/I children did not demonstrate cue dependency effects on the CRT task, suggesting that they were failing to incorporate relevant information before making a response. The sluggish and inhibited performance of the ADHD/I group challenges the idea that it is a subtype of ADHD. Key words: ADHD; subtypes; disinhibition; sluggish cognitive tempo
\end{abstract}


Response Style Differences in the Inattentive and Combined Subtypes of AttentionDeficit/Hyperactivity Disorder

Within the last 20 years, the ADHD research community has amassed an impressive body of work characterizing the nature of the disorder, including deficient inhibitory functioning and problems in working memory (Barkley, 2006; Hartung, Milich, Lynam \& Martin, 2002; Nigg, 2001; Pennington \& Ozonoff, 1996). Additionally, recent technological advances have allowed researchers to probe genetic and physiological underpinnings of the disorder (Barr et al., 2000; Daly, Hawi, Fitzgerald, \& Gill, 1999), providing additional evidence with which to build sound models for etiology and pathology. The conclusions drawn from this literature are nearly always limited, however, to describing only the phenomenology of the combined type of ADHD (ADHD/C), while the inattentive subtype of attention-deficit/hyperactivity disorder (ADHD/I) remains surprisingly understudied (Barkley, 1997; Diamond, 2005; Milich, Balentine, \& Lynam, 2001; Nigg, 2006). Although this trend is changing, it is still unclear whether currently accepted models of the nature of ADHD (e.g., Barkley, 1997; Nigg, 2001) are common across ADHD subtypes or apply only to ADHD/C.

\section{ADHD Subtypes}

The DSM-IV (American Psychiatric Association, 1994) describes ADHD as a heterogeneous disorder, providing diagnostic criteria for three subtypes: primarily hyperactive/impulsive (ADHD/HI), primarily inattentive (ADHD/I), and combined type (ADHD/C; APA, 1994). ADHD/HI is thought to be relatively rare and is often conceptualized as a pre-cursor to ADHD/C (Lahey, Pelham, Loney, Lee, \& Willcutt, 2005). Further, relatively little empirical evidence is available supporting the validity of this group (Faraone, Biederman, 
Weber, \& Russell, 1998; Milich et al., 2001). Thus, when subtypes of ADHD are considered, attention is usually directed to the $\mathrm{ADHD} / \mathrm{C}$ and $\mathrm{ADHD} / \mathrm{I}$ groups.

According to the DSM-IV, the inattentive subtype of ADHD shares all of the symptoms of inattention with the combined subtype but lacks clinically significant hyperactive and impulsive behaviors (APA, 1994). A current controversy in the field, however, concerns whether $\mathrm{ADHD} / \mathrm{I}$ is better conceptualized as a distinct disorder, rather than as a related subtype or less extreme variant of ADHD (Barkley, 2001; Diamond, 2005; Milich et al., 2001; Solanto et al., 2007; but see also Baeyens, Roeyers, \& Vande Walle, 2006; Hinshaw, 2001; Lahey, 2001; Pelham, 2001). Indeed, recent research suggests that ADHD/I and ADHD/C differ on several important dimensions other than the presence of hyperactive and impulsive symptoms (e.g., Bauermeister, Barkley, \& Martinez, 2005; Carlson \& Mann, 2000; Riccio, Homack, JarrattPizzitola, \& Wolfe, 2006).

For instance, ADHD/I has been associated with a later age of onset (Faraone et al., 1998), later age of referral (McBurnett et al, 1999), lower mathematics achievement (Marshall, Hynd, Handwerk, \& Hall, 1997), slower processing speed (Solanto et al., 2007), and different genetic profiles than ADHD/C (Rowe, Stever, \& Gard, 1998; Smoller et al., 2006). Additionally, in contrast to the disruptiveness and disinhibition associated with ADHD/C, those with ADHD/I have been described as hypoactive, easily bored, self-conscious, unmotivated, and shy (Hinshaw, 2002; Maedgen \& Carlson, 2000). Further, the actual attention problems exhibited by those children with ADHD/I appear to be different from those found in children with ADHD/C. While $\mathrm{ADHD} / \mathrm{C}$ inattention is often associated with distractibility, ADHD/I inattention seems to be characterized by a "sluggish cognitive tempo," marked by drowsiness, lethargy, and passivity (Bauermeister et al., 2005; Carlson \& Mann, 2000; Milich et al., 2001), although these 
distinctions have yet to be demonstrated behaviorally (Solanto et al., 2007).

Consistent with these differences in symptom profile, children with ADHD/I demonstrate different associated features. These children are less likely to have comorbid conduct problems and more likely to have comorbid internalizing disorders than children with ADHD/C (Nigg, 2000; Weiss, Worling, \& Wasdell; 2003). Children with ADHD/I also may be less likely to respond to methylphenidate treatment (Barkley, 2001; O’Driscoll et al., 2005; but see also Wilens et al., 2003), and the two groups show differential types of social deficits, with ADHD/C children eliciting more social rejection, and ADHD/I children eliciting more social neglect from their peers (Maedgen \& Carlson, 2000).

Although the results from these studies suggest important differences between the $\mathrm{ADHD} / \mathrm{C}$ and ADHD/I groups, these studies are relatively small in number. Even among the available studies, many are further limited by their inclusion of inadequately defined subtype groups. As Milich et al. (2001) point out, the DSM-IV criteria allow ADHD/I to be conceptualized as subthreshold ADHD/C; that is, children who have several hyperactive and impulsive symptoms but not enough for a diagnosis of ADHD/C are diagnosed with the inattentive subtype. Variation in the definition of ADHD/I results in heterogeneous samples of these individuals, ranging from those with clinically significant inattentive symptoms but no hyperactive/impulsive behaviors, to those with similar inattentive symptoms and substantial but subthreshold hyperactive/impulsive behaviors. This contamination of groups yields results that are difficult to reconcile with the existing literature. Milich and colleagues (2001) have recommended that future work utilize clear delineation when examining the ADHD/I group so that potential differences among the subtypes of this diagnostic category can be better understood. 


\section{Response Inhibition in ADHD}

A growing consensus is emerging that ADHD is characterized by deficits in inhibitory functioning (Barkley, 1997; Newman \& Wallace, 1993; Nigg, 2001; Pennington \& Ozonoff, 1996). Substantial evidence from the recent ADHD literature has focused heavily on describing the role these inhibitory deficits play in the etiology and pathology of the disorder (Barkley, 1997; Castellanos \& Tannock, 2002; Nigg, 2001, 2006; Quay 1988, 1997). However, several authors acknowledge that conclusions regarding response inhibition in ADHD must be restricted to ADHD/C due to the paucity of research on inhibitory functioning in individuals with ADHD/I (e.g., Barkley, 1997; Nigg, 2001, 2006).

Defined broadly, inhibition is the process of suppressing an inappropriate response. According to Barkley's (1997) influential model, deficits in response inhibition are the primary underlying feature of ADHD, and have downstream effects resulting in the characteristic behavioral and academic impairments observed in ADHD. Other theorists have divided the construct of response inhibition into components, including executive inhibition, which involves the effortful suppression of a response in order to pursue some goal (Barkley, 1997; Logan, 1994; Nigg, 2001), and motivational inhibition, which refers to the suppression of a response in the face of negative consequences or emotionally aversive feedback (Logan, 1994; Nigg, 2001; Quay, 1997). Although less evidence is available regarding the nature of motivational inhibition in ADHD (see Nigg, 2006, for a review), there is substantial empirical support for executive inhibitory deficits in ADHD for both children and adults (Carr, Nigg, \& Henderson, 2006; Epstein, Johnson, Varia, \& Conners, 2001; Lijffijt, Kenemans, Verbaten, \& van Engeland, 2005; Logan \& Irwin, 2000; Nigg, 2006; Nigg, Blaskey, Huang-Pollock, \& Rappley, 2002).

\section{Executive Inhibition}


A variety of assessment tools have been developed over the years to measure executive inhibition (Nichols \& Waschbusch, 2004; also see Nigg, 2001). Results of numerous investigations suggest that executive deficits are highly associated with ADHD, as evidenced by greater rates of failed inhibitory responses (Barkley, 1997; Pennington \& Ozonoff, 1996), although other factors typically associated with ADHD, such as comorbid anxiety, have been found to attenuate these findings (Pliszka, Borcherding, Spratley, Leon, \& Irick, 1997). Intuitively, deficient inhibitory behavior might be more closely linked to the hyperactiveimpulsive symptoms common to $\mathrm{ADHD} / \mathrm{C}$ rather than the inattentive symptoms shared between the subtypes. Supporting this hypothesis, boys with ADHD/C have been shown to demonstrate greater impairment in inhibiting responses, as measured by an antisaccade task, relative to boys with ADHD/I (O’Driscoll, et al., 2005). Specifically, O’Driscoll and colleagues found that while boys with ADHD/C differed significantly from healthy comparison children on measures of inhibitory failure, boys with ADHD/I did not (2005). These findings suggest a stronger link between problems in executive inhibitory behavior for ADHD/C than ADHD/I.

Although the majority of ADHD research has employed standard behavioral inhibition tasks, such as the stop task (Lijffijt et al., 2005; Logan, 1994; Logan \& Cowan, 1984; Oosterlaan, Logan, \& Sergeant, 1998), other types of inhibition tasks have begun to be utilized. The cued reaction time task (CRT) allows researchers to study the specific role of prepotency and anticipatory mechanisms of control in the execution and inhibition of behavior by manipulating cue-target pairings. Valid cues tend to facilitate response execution and inhibition, although the nature of these relations may change as a function of environmental manipulations, such as exposure to alcohol (Marczinski \& Fillmore, 2003; Abroms, Fillmore, \& Marczinski, 2003; Fillmore, Marczinski, \& Bowman, 2005). 
With respect to ADHD, only one study has demonstrated cue-related deficits among children with ADHD as compared to children without the disorder (Durston et al., 2007). Specifically, these authors used a modified go/no-go inhibition task where predictability of stimuli was manipulated to develop expectancies in the participants. On trials where the gostimuli were predictable, comparison children exhibited faster reaction times. Children with ADHD, however, did not exhibit this improved performance to the same degree. Thus, it appears that children with ADHD may experience difficulty interpreting cue-related information on tasks of cognitive control. However, it is important to note that the authors did not delineate between ADHD subtypes in their study. It is therefore unclear whether these findings would apply to children with ADHD/C, ADHD/I, or both subtypes.

\section{Motivational Inhibition}

A related form of inhibition reflects individuals' tendency to activate or inhibit behavioral responses based on the presence of reinforcing or punishing cues in the environment (Gray, 1991; Nigg, 2006). In this motivational model, behavioral inhibition occurs in response to some anxiety- or fear-inducing stimulus that causes the individual to abandon the prepotent response. Quay $(1988,1997)$ argued that deficits in Gray's conceptual behavioral inhibition system (BIS; i.e., discontinue or withhold previously rewarded responding following punishment) are an integral piece in the ADHD puzzle. However, this "weak BIS" model has received mixed empirical support (e.g., Iaboni, Douglas, \& Baker, 1995; Iaboni, Douglas, \& Ditto, 1997; Oosterlaan et al., 1998; Pliszka, Hatch, Bocherding, \& Rogeness, 1993), leading Newman and Wallace (1993) to develop a response modulation model that incorporates Gray's behavioral activation system (BAS; i.e., approach or respond following rewarding feedback) in addition to BIS. The response modulation hypothesis proposes that individuals are constantly evaluating 
feedback from the environment to inform their behavior. For individuals with a response modulation deficit, if most of this feedback is perceived as rewarding or salient, behavior continues unchanged (i.e., strong BAS activation), despite contradictory feedback (i.e., weak BIS activation). Thus, according to the response modulation hypothesis, behavioral disinhibition results not only from weak BIS activity, but also from dominant BAS activity that effectively “overrides” BIS activity (MacCoon, Wallace, \& Newman, 2004; Patterson \& Newman, 1993).

Response modulation is assessed by tasks such as a mixed contingency go/no-go task (GNG; Newman \& Wallace, 1993), where correct responses elicit rewarding feedback but incorrect responses result in negative consequences for the participant. The response modulation hypothesis suggests that rewarding feedback will be more influential in driving response patterns for individuals with ADHD/C, such that they will make more commission errors (i.e., failure to inhibit to no-go trials) than healthy comparison children. While some have demonstrated this pattern to some degree (e.g., Hartung et al., 2002; Iaboni, Douglas, \& Baker, 1995; Milich, Hartung, Martin \& Haigler, 1994), empirical support for the response modulation hypothesis in ADHD is mixed in the ADHD/C literature (Luman, Oosterlaan, \& Sergeant, 2005; Nigg, 2006).

\section{The Current Study}

At this point, it remains unclear how well contemporary models regarding the inhibitory deficits of ADHD generalize across the subtypes of the disorder. The purpose of the current study is to use clear, categorical criteria to identify individuals with ADHD/I and ADHD/C, as well as a group of healthy comparison peers, and compare their performance on tasks measuring inhibitory functioning. Two separate tasks were selected to provide information regarding basic response variables such as reaction time, as well as the degree to which inhibitory behavior may be impaired as a function of motivational contingencies. The cued-reaction-time task was 
included as a measure of basic inhibitory control, whereas the go/no-go incorporated reward and punishment motivational contingencies, thus assessing a more complex inhibitory process.

Based on previous findings (Hartung, et al., 2002; Milich et al, 1994), we predicted that the ADHD/C group would exhibit deficits in inhibiting behavior during an ongoing task with competing reward and punishment contingencies (i.e., more commission errors than others). Previous studies suggesting the association between ADHD/I and sluggish cognitive tempo (e.g., Carlson \& Mann, 2002), working memory deficits (Barkley, 2006, Diamond, 2005), and processing speed (Solanto et al., 2007) led us to hypothesize that the ADHD/I group would respond more slowly to cues across both tasks, demonstrating few deficits in inhibiting behavior. Method

\section{Participants}

A sample of 54 children with ADHD and 21 comparison children participated in this study. The children in each diagnostic group were between the ages of 9 and 12 years $(M$ age $=$ 10.86 years, $S D=1.09$ ). Approximately $85 \%$ of the children were Caucasian, $11 \%$ were African American and 4\% identified themselves as other. The children with ADHD were recruited from the Hyperactive Children's Clinic in the School of Medicine at the University of Kentucky. The children were carefully selected to fulfill the DSM-IV criteria for either the ADHD inattentive subtype or ADHD combined subtype and to be free of confounding factors (i.e., low IQ, neurological problems). Diagnoses were based on a convergence of evidence from multiple informants and multiple measures.

Children were first required to have received the appropriate DSM-IV ADHD diagnosis (i.e., inattentive or combined) based on a comprehensive psychiatric clinic evaluation at the Hyperactive Children's Clinic. This evaluation utilizes multiple assessment procedures designed 
to identify psychiatric and neurological factors that may influence or better account for ADHD symptoms, such as mood disorders, developmental disorders, epilepsy or neurological disorders, or mental retardation. In addition, the medical charts of all the children were reviewed in detail to gather specific information about ADHD diagnoses and medical history, including reason for clinic referral, age at onset of symptoms, classroom behavior via teacher ratings, evidence of impairment, parent ratings of behavior, IQ, medical history, and comorbid diagnoses. Children were excluded from the study if their medical charts provided evidence of IQ scores lower than 80, presence of sensory impairments, diagnoses of epilepsy or other neurological disorders, or prescribed medication that could not be temporarily discontinued during study participation. Further, children who were diagnosed by the Hyperactive Children's Clinic with ADHD primarily hyperactive/impulsive subtype were excluded from the study.

If the above criteria were met, then a parent of the child with ADHD was contacted and invited to participate in the study. During the testing session, a semi-structured interview, similar to the P-ChIPS (Weller, Weller, Rooney, \& Fristad, 1999), but only consisting of verbatim DSMIV criteria for ADHD and ODD, was conducted with the parent to confirm the child's diagnostic status. This interview also provided a common measure of ADHD symptoms for all children in the study, because the assessment measures used to make the initial clinic diagnosis varied. The interviews were conducted by trained graduate students in clinical psychology. In the interview, the parent was asked whether each diagnostic criterion was true of his/her child, and, if so, the parent was asked to give behavioral examples. If a behavior was characteristic of the child, the parent was additionally asked whether that behavior seemed inappropriate for the child's age and whether it impaired the child's functioning academically and/or socially. A diagnostic criterion was considered to be endorsed only if the parent indicated the behavior was age inappropriate 
and impairing. This interview procedure has been used successfully by our research group in previous studies, with interrater reliabilities for the number of ADHD symptoms endorsed by the parent above $95 \%$ (e.g., Lorch et al., 1999). The data for children whose parental interviews supported an ADHD diagnosis were retained for analysis in this study. In addition to the information from the structured psychiatric interview, parents completed the Child Behavior Checklist (Achenbach, 1991) and the Conners (1997) Parent Rating Scales (CPRS-R:S).

Diagnostic Subgroups. Children with ADHD were assigned to one of the two subgroups under investigation (ADHD/I; ADHD/C) based on the history obtained from the medical chart review as well as the more systematic data obtained from the structured interview and the CBCL and CPRS-R:S. Consistent with the exclusion criteria utilized at the first stage of recruitment, no children with ADHD hyperactive/impulsive subtype were identified within this group. To be placed in the ADHD/C group ( $n=37)$, children must have met criteria for this diagnosis on the structured interview, and have $T$ scores above 60 on the Conners Hyperactivity scale and the ADHD Index. Consistent with the literature suggesting a common comorbidity between ADHD/C and oppositional behavior (Weiss et al., 2003; Nigg, 2000), children demonstrating clinically significant conduct problems were not excluded from the ADHD/C group. In forming the ADHD/I group $(n=17)$, recommendations made by Milich et al. (2001) were followed to ensure that this group did not include subthreshold ADHD/C children. Thus, the children in the $\mathrm{ADHD} / \mathrm{I}$ group were required to meet criteria for attention problems on the structured interview and have 3 or fewer symptoms on the hyperactive/impulsive dimension. In addition, the children were required to have $T$ scores above 60 on the Conners Cognitive Problems/Inattention scale and $T$ scores less than 60 on the Hyperactivity scale. Finally, the children in the ADHD/I group were required to have $T$ scores below 60 on the CBCL Aggression and Delinquency scales. 
Table 1 summarizes the mean scores on these diagnostic measures for the two ADHD groups, documenting that the groups differed significantly on the relevant diagnostic indicators. Due to potential impact of comorbid anxiety on behavioral performance (Nigg, 2001), Table 1 also provides mean CBCL Anxiety scale scores for the groups. There were no significant differences in anxiety scores among groups.

The comparison group of children without ADHD was recruited through newspaper advertisements, posted advertisements in the community, and by word-of-mouth. They were screened during a recruitment phone call in which the parents were asked if their child had ever been referred for any behavioral or learning problems. The comparison children were not required to be symptom free, but had to have two or fewer symptoms in a diagnostic category. These children were significantly less symptomatic than the children with ADHD in terms of the $D S M-I V$ criteria for inattentive symptoms and hyperactivity symptoms, and moreover did not meet diagnostic criteria for any subtype of ADHD. In addition, the children in the comparison group had to have $T$ scores below 60 on all of the relevant rating scales. As indicated in Table 1, the diagnostic interview and rating scale data successfully differentiated between the comparison children and the two ADHD groups.

Among the children with ADHD, 34 of the 54 children (63\%) were being treated with psychostimulant medication. The remaining 41 children who participated in this study were not taking any prescribed medication. No child received any psychostimulant medication on the day of the study until after the session was completed. This provided a sufficient time period (approximately 24 hours) for clearance of any medication administered on the day before the session. Participants who were receiving other medications that could not be easily withdrawn for testing (e.g., chlonidine) were excluded at the time of enrollment. All children received two 
small toys and $\$ 30.00$ for their participation in the study. Groups were not significantly different on the basis of age, gender, racial composition, grade level, maternal education, paternal education, or KBIT vocabulary scores (see Table 2). Significant differences between groups were observed, however, for KBIT matrices scores, $F(2,74)=5.15, p=.008$, with comparison children receiving higher scores than both ADHD groups. Diagnostic group comparison (ADHD/I vs. ADHD/C) did not evidence any significant differences between these two groups in terms of age, gender, racial composition, grade level, maternal education, paternal education, KBIT vocabulary scores, or KBIT matrices scores.

\section{Procedure}

The study took place at the Behavioral Pharmacology and Neurocognition Research Laboratory in the Department of Psychology at the University of Kentucky. All children were tested individually. Testing occurred on a non-school day between the hours of 9 am and 5 pm. Upon arrival at the lab the child and parent were greeted by two experimenters who described the general details of the study and the basic testing procedures. Written consent was then obtained from the parent and verbal assent was obtained from the child. After obtaining consent, one of the experimenters accompanied the parent to an interview room to complete the semi-structured interview and questionnaires. The other experimenter accompanied the child to a nearby testing room to complete the testing. The administration of these tasks was part of a larger testing battery that contained neuropsychological tests and other measures of cognitive functioning. Prior to each test, the experimenter provided the task instructions and the children performed a brief 2-3 min familiarization test on each task to ensure that the child understood the task requirements. Children were given a 15-minute break between each testing component. At the conclusion of the session, the child and parent were debriefed and paid for their participation. 


\section{Measures}

Cued-reaction-time task (CRT). The cued-reaction-time task (CRT) was included as a measure of basic inhibitory functioning. In this task, participants were required to respond to go target stimuli and withhold responding to no-go target stimuli. Each target was preceded by either a go cue or a no-go cue, such that the go target was preceded by the go cue during $80 \%$ of the trials, and by the no-go cue during the remaining 20\%; conversely, no-go targets were preceded by the no-go cue $80 \%$ of the time and by the go cue the remaining $20 \%$. Cues were 7.5 $\mathrm{cm} \times 2.5 \mathrm{~cm}$ rectangles framed in $0.8 \mathrm{~mm}$ black outlines presented against a white background on a 17 " computer monitor. Cues were presented in one of two orientations: vertical (height $=$ $7.5 \mathrm{~cm}$, width $=2.5 \mathrm{~cm}$ ) or horizontal (height $=2.5 \mathrm{~cm}$, width $=7.5 \mathrm{~cm})$. The green and blue targets were displayed on the monitor as solid hues that filled the interior of the rectangle. Participants were instructed to press the forward slash (/) key on the keyboard when a green (go) target was presented and to inhibit any response when a blue (no-go) target occurred. Key presses were made with the index finger of the preferred hand. The vertical cue preceded the gotarget (green) on $80 \%$ of trials and preceded the no-go target (blue) on $20 \%$ of trials. The horizontal cue preceded the go-target on $20 \%$ of trials and preceded the no-go target on $80 \%$ of trials. Thus, based on these cue-target pairings, vertical and horizontal cues operated as go and no-go cues, respectively.

A trial began with the presentation of a fixation point (+) for $800 \mathrm{~ms}$, followed by a blank, white screen for $500 \mathrm{~ms}$. The cue was then displayed for one of five stimulus onset asynchronies (SOAs $=100,200,300,400$, and $500 \mathrm{~ms}$ ), before a go or no-go target was displayed. Targets remained visible for $1000 \mathrm{~ms}$ or were terminated once a response occurred. When a response to a go target occurred, the screen displayed the amount of time it took to make 
that response in milliseconds. The word "incorrect" was displayed following responses to no-go targets. This screen was displayed for $700 \mathrm{~ms}$ before the start of the next trial. A test consisted of 150 trials with an equal number of go and no-go targets (i.e., 75), and an equal number of vertical and horizontal cues (i.e., 75). There were four possible cue-target combinations. The five SOAs were presented equally often (30 times) during a test, and an equal number of SOAs separated each cue-target combination. The presentation order of cue-target combinations and SOAs was random. On each test trial the computer recorded the subject in milliseconds from the onset of the target display until the key was pressed. The accuracy of the response was also recorded. The test required 8 minutes to complete. Participants were screened to ensure they could discriminate between blue and green prior to the task. The dependent variables of interest recorded from this task were proportion of inhibitory failures following go cues (incorrect key press to no-go target following go cue), proportion of inhibitory failures following no-go cues (incorrect key press to no-go target following no-go cue), reaction time following go cue, reaction time following no-go cue, reaction time standard deviation following go cue, and reaction time standard deviation following no-go cue. Omission errors to go-targets on this task are also recorded but are typically infrequent (e.g., less than 2-3 errors per test on average), precluding any meaningful statistical analyses. This was also evident in the present study, in that less than $3 \%$ of the trials results in omission errors on average during the test.

Go/no-go task (GNG). The go/no-go task (GNG) was used as a measure of inhibitory functioning with competing motivational contingencies. The task employed a mixed contingency feedback schedule, meaning that both rewarding and punishing feedback were used following participants' responses-here, monetary gain or loss, respectively_as opposed to either rewards or punishments alone. This task required participants to learn via trial and error when to respond 
and when to inhibit responding based on feedback. Stimuli included eight, 2-digit numbers presented individually on a computer monitor. The white numbers were presented on a black background and ranged in size from 3-4 $\mathrm{cm}$ wide and 2-3 cm tall. Stimuli remained on the screen for $3 \mathrm{sec}$ or until a response was made. Participants were instructed to press the (/) key with the index finger of the preferred hand to "good numbers" (i.e., go stimuli; 15, 42, 74, 96) and not to press to "bad numbers" (i.e., no-go stimuli; $21,38,57,84$ ). The task started with a pre-treatment to initiate responding by introducing the 4 go stimuli. All 8 numbers were then presented randomly within each of 10 blocks, with each number presented once per block, resulting in 84 total trials. Feedback was presented following each key press. If the participant pressed the key to a go stimulus, a cash register sound effect played over the speakers and a message reading, "you won 5 cents," was displayed. If the participant responded to a no-go stimulus, the participant would hear a buzzer and the message, "you lost 5 cents," was displayed. The feedback was displayed for $750 \mathrm{~ms}$ and was followed by a blank screen for 1 second before the next number appeared. If the participant did not press, then the number would disappear and the screen would be blank for 1 second before the next trial. In addition to visual and audio feedback from the computer, standard poker chips representing 5 cents each were placed in front of the participants so they could track how much money they had won or lost. Participants started with 10 chips. Each time the participant correctly responded to a go trial, the experimenter added a chip to the stack; each time the participant inappropriately responded to a no-go trial (i.e., commission error), the experimenter removed a chip. The dependent variables of interest recorded from this task were number of omission errors (failures to respond to go stimuli) out of 40, number of commission errors (responding to no-go stimuli) out of 40, average reaction time, and reaction time standard deviation. This task required approximately 7 minutes to complete. 


\section{Results}

Results for CRT task performance can be found in Table 3. Interestingly, group differences were not supported for CRT proportion of inhibitory failures following the go cue, $F$ $(2,74)=1.91, n s$, or CRT proportion of inhibitory failures following the no-go cue, $F(2,74)=$ 0.58, ns. Paired samples t-tests were conducted to assess whether groups demonstrated withingroup cue-dependency, or differed in the number of inhibitory failures following valid and invalid cues. Specifically, cue-dependency effects suggest that more inhibitory failures (i.e., responding to a no-go target) should occur following an invalid (i.e., go) cue, as this cue incorrectly signals to the participant that the following stimulus will be a go target, thereby priming a go response. In contrast, fewer inhibitory failures should follow a valid (i.e., no-go) cue, as this cue correctly signals the participant of the impending no-go target. Due to this cuedependency effect, participants should have significantly more errors following the go cues than the no-go cues. Consistent with this prediction, significant differences were found for the comparison $t(20)=2.97, p<.01, d=1.33$, and ADHD/C, $t(36)=3.44, p<.01, d=1.15$ groups, suggesting that their subsequent behavior was consistent with the priming of the preceding cue. However, this difference in errors following the different cues was not significant for the $\mathrm{ADHD} / \mathrm{I}$ group, $t(16)=.48, n s$, suggesting that the ADHD/I group was making similar numbers of inhibitory failures regardless of the validity of the preceding cue (see Figure 1, left panel).

With regard to CRT reaction time and reaction time variability, ANOVAs revealed significant group differences for CRT reaction times following go cues, $F(2,74)=5.17, p<.01$, and following no-go cues, $F(2,74)=4.20, p<.01$. Planned comparisons demonstrated that the ADHD/I group demonstrated slower CRT reaction times following go cues than the comparison $t(36)=4.08, p<.001, d=1.36$, and ADHD/C $t(52)=2.10, p<.05, d=.58$, groups (see Figure 
1, right panel). For reaction times following no-go cues, the ADHD/I group again exhibited slower reaction times than the comparison group, $t(36)=3.75, p<.01, d=1.25$, but not the ADHD/C group, $t(52)=.14, n s, d=.04$ (see Figure 1, right panel). Significant differences were also found among groups for the CRT reaction time standard deviation following no-go cues, $F$ $(2,74)=4.10, p<.05$, although the reaction time standard deviation following go cues was not significantly different across groups, $F(2,74)=1.35, n s$. Planned comparisons demonstrated that both ADHD subgroups exhibited more variable CRT reaction times following no-go cues than the comparison group, $t(36)=2.55, p<.05, d=.85$, and $t(56)=2.78, p<.01, d=.74$, for ADHD/I, and ADHD/C groups, respectively.

Results for GNG task performance can be found in Table 4. ANOVAs revealed significant group differences for GNG errors of omission, $F(2,74)=5.81, p<.01$. Planned comparisons demonstrated that the ADHD/I group exhibited more GNG errors of omission than the comparison $t(36)=2.31, p<.05, d=.77$, and ADHD/C $t(52)=3.32, p<.01, d=.92$, groups. Contrary to hypotheses, no group differences were found for GNG errors of commission, $F$ (2, 74) $=0.85, n s$.

In addition to higher numbers of GNG omission errors, the ADHD/I subtype demonstrated other results theoretically consistent with a slow cognitive tempo. ANOVAs revealed significant group differences for GNG average reaction time, $F(2,74)=5.93, p<.01$, and GNG reaction time standard deviation, $F(2,74)=4.44, p<.05$. Planned comparisons demonstrated that ADHD/I participants had slower response times to GNG stimuli than the comparison $t(36)=2.80, p<.01, d=.93$, and $\mathrm{ADHD} / \mathrm{C} t(52)=3.31, p<.01, d=.92$, groups, and that $\mathrm{ADHD} / \mathrm{I}$ participants' reaction times were significantly more variable than the comparison $t(36)=2.83, p<.01, d=.94$, and ADHD/C $t(52)=2.37, p<.05, d=.66$, groups. 
Thus, the ADHD/I group appeared to respond much more slowly and with more variation in reaction times than the other two groups during the GNG task.

Finally, due to group differences on KBIT matrices scores, we conducted univariate analyses using KBIT matrices scores as a covariate. Analyses of covariance revealed that four dependent measures continued to show significance: CRT reaction times following go cues, $F$ (2, $74)=4.53, p<.05$, GNG errors of omission, $F(2,74)=5.75, p<.01$; GNG average reaction time, $F(2,74)=5.63, p<.01$, and GNG reaction time standard deviation, $F(2,74)=4.05, p<$ .05. However, two of the previously significant relations became marginally significant: CRT reaction time following no-go cues, $F(2,74)=3.01, p<.06$; and CRT reaction time standard deviation following no-go cues, $F(2,74)=2.75, p<.07$. Thus, although many of the findings appeared resilient to the effects of this aspect of intelligence, control of KBIT matrices slightly attenuated the effect of ADHD/I symptoms on reaction time following no-go cues, and ADHD/I and $\mathrm{ADHD} / \mathrm{C}$ symptoms on reaction time variability following no-go cues on the CRT task.

\section{Discussion}

In this study, we examined the performance of children meeting criteria for the two ADHD subtypes, $\mathrm{ADHD} / \mathrm{I}$ and $\mathrm{ADHD} / \mathrm{C}$, as well as that of comparison children, on two tasks of behavioral inhibition. This study extends the previous line of research regarding potential etiological and behavioral differences between the ADHD subtypes, and lends support to the idea that these subtypes are distinct disorders. Performance on a task of basic inhibitory functioning and a more complex task involving motivational contingencies revealed clear differences between the ADHD/I group and the other two groups.

First, the ADHD/I group responded to stimuli in ways that are consistent with previous descriptions of a sluggish cognitive tempo (Carlson \& Mann, 2002; Hartman, Willcutt, Rhee, \& 
Pennington, 2004; Milich et al., 2001). Rather than quick, impulsive responding, the ADHD/I group demonstrated slow and variable attention in responding to targets on the CRT task, a basic inhibitory task, as well as in responding to targets on the GNG task, a motivational inhibition task. This consistently slow response style across tasks suggests that this sluggish cognitive tempo is pervasive and not contingent on any specific aspect of the selected tasks. Additionally, while both ADHD/I and ADHD/C groups demonstrated high CRT response-time variability following no-go cues, only the ADHD/I group had notably high response-time variability during the GNG task. Such RT variability is considered a measure of difficulty maintaining attention (Castellanos \& Tannock, 2002). Thus, although both groups exhibited problems sustaining attention, only the ADHD/I group showed consistent inattention across contexts, which is consistent with the hypothesis that the ADHD/I group is characterized by a sluggish cognitive style.

Further, only those with ADHD/I failed to demonstrate a cue-dependency effect in the CRT task. This is an important divergence, given that both the comparison and ADHD/C children demonstrated the typical cue-dependency scores, illustrated by significantly greater inhibitory failures following CRT go than no-go cues. Cue-dependency is considered a rather resilient effect that continues to be evident despite behavioral inhibition deficits. For example, previous research using alcohol administration suggests that although increased blood alcohol content leads to increases in inhibitory failures, it has limited impact on cue dependency (Abroms et al., 2003; Fillmore et al., 2005). Thus, the similar responding of the ADHD/I children following both valid and invalid cue conditions indicates that ADHD/I children are strikingly unable to utilize relevant environmental cues in their responding.

Interestingly, the slower reaction time of the ADHD/I group did little to improve 
performance. The children with ADHD/I did not make significantly fewer CRT inhibitory failures or GNG commission errors than either ADHD/C or comparison peers. In fact, children with ADHD/I responded less frequently to rewarded targets in the GNG task (i.e., higher errors of omission). While failures to respond to GNG stimuli might be interpreted as a cautious or deliberate response style, it is important to note that the GNG task required a response within 3 seconds of stimulus presentation. Given that the ADHD/I group responded more slowly to all targets, it may be that these errors of omission are associated in part with slower reaction times overall (i.e., not responding within the 3 -second time limit).

To ensure that these group differences could not be better accounted for by confounding factors, we identified other variables that may have influenced results. Although the ADHD/I group's performance suggests an overly inhibited style, failure to find between group differences on CBCL anxiety scores suggested that this style is likely not better accounted for by comorbid internalizing pathology. Similarly, due to group differences in KBIT matrices scores, we included these as a statistical control. While control of KBIT matrices did slightly attenuate two of these relations, the majority of the study findings were largely unchanged, suggesting that these group differences in performance and reaction time are not simply a product of group differences in performance IQ scores.

Taken together, these results suggest that the children with ADHD/I demonstrate a dramatically different response style from those with ADHD/C; that is, the ADHD/I group appears to be distinguishable by a slow, variable, and perhaps even cautious style. This response style is inconsistent with what is known about the ADHD/C subtype, and supports the argument that these two subtypes are distinct disorders with unique phenotypes and underlying pathologies (Diamond, 2005; Barkley, 2001; Milich et al., 2001; O’Driscoll et al., 2005) 
The use of two different types of tasks in this study allowed further exploration of the impact of motivational contingencies on the performance of individuals with ADHD/I. The slow and variable performance of the ADHD/I group across both the simple executive (CRT) and more complex motivational inhibitory (GNG) tasks does not appear to support assertions that the ADHD/I subtype is associated with a motivational deficit (Diamond, 2005). Specifically, Diamond (2005) states that the primary problem for children with ADHD/I is one of motivation rather than disinhibition, and therefore motivational contingencies may enhance performance of this group. Of this Diamond writes, "challenge or risk, something to literally get their adrenaline pumping, can be key to getting their attention, and to eliciting optimum performance" (2005; $\mathrm{p}$. 810). In this study, the failure of motivational contingencies (e.g., earning and losing money on the GNG task) to enhance or even change the sluggish performance of those in the ADHD/I group does not appear to support this hypothesis.

However, it is possible that the incentives employed in the present study were not sufficient to arouse the children with $\mathrm{ADHD} / \mathrm{I}$ to respond. In fact, rather than interpreting the results for the ADHD/I group as indicative of a sluggish cognitive tempo, it is possible that the present findings reflect an overly cautious and inhibited response style, one in which these children do not respond when uncertain about the correctness of their response. Recent work by Huang-Pollock, Mikami, Pfiffner, and McBurnett (2007) suggests that motivational incentives can decrease inhibitory problems in ADHD/I children when contingencies are placed in appropriate context, such as increasing incentives over time. While no inhibitory deficits were found for this group in the present study, it is possible that increasing incentives would provide the ADHD/I children more motivation to respond. However, increasing incentives over time also allows for additional processing and habituation to the task at hand, and thus does not 
conclusively rule out discrete cognitive processing deficits. Further, it is also necessary to consider the possibility that sluggish cognitive tempo and inhibited response style are not mutually exclusive, and the children with ADHD/I may be both cautious and slow information processors. In the future, investigators may want to design measures that are more suited to distinguishing between these two interpretations-slow responses owing to slowed informative processing versus a deliberately cautious approach to the task.

Consistent with arguments regarding a primary importance of deficits in sustaining attention to the construct of ADHD (Castellanos, Sonuga-Barke, Milham, \& Tannock, 2006; Castellanos \& Tannock, 2002), both the ADHD/C and ADHD/I groups demonstrated significantly increased reaction time variability than comparison children in the CRT task following no-go cues. This inability to sustain attention from trial to trial has been defined by Castellanos and Tannock to be one of the core defining features of ADHD, and is consistent with the diagnostic criteria of ADHD from the DSM-IV. Consistent with this hypothesis, a recent study found increased variability on reaction time trials to be the most influential mediator of story comprehension deficits among children with ADHD/C (Flory et al., 2006), reflecting its importance as a core deficit of the disorder.

In contrast to these notable deficits in attention, the ADHD/C group did not demonstrate the predicted behavioral inhibition deficits in the CRT and GNG tasks. Although these findings appear difficult to reconcile with models positing inhibitory dysfunction as the core deficit of ADHD (Barkley, 1997), others have also noted such inconsistencies with specific behavioral paradigms (Rommelse et al., 2007). It is therefore possible that the current findings reflect shortcomings of the tasks employed to investigate differential aspects of inhibitory functioning.

The CRT task utilizes a very basic structure designed to assess the impact of cues on 
target response. While inhibitory performance is an important aspect of this design, the simplicity of the task often results in little variability in response; the majority of participants respond appropriately to targets. Given this, this CRT task may not have been adequately complex to activate higher-order behavioral deficits. This is consistent with Rommelse et al. (2007), who have recently suggested that for those with ADHD, higher order inhibition deficits may stem from lower order cognitive difficulties, such as problems in encoding, processing, and perceiving stimuli. Thus, inhibitory failures not be evident in tasks that demand little from lower order processes, such as the CRT (Rommelse et al., 2007).

In contrast to the basic design of the CRT, the GNG is a complex task that has relatively higher demand on cognitive resources. Successful performance depends upon behavioral inhibition, memory of the stimuli presented, reaction time constraints, and motivation. Although the ADHD/C participants did demonstrate modestly higher errors of commission on the GNG task than the both the ADHD/I and comparison children, differences were not significant. This failure to replicate significant findings in past research is perhaps understandable, however, given the previous GNG task findings. Relations between ADHD/C and increased GNG commission errors have been found in only three studies, and for these studies, results were complex (Hartung et al., 2002; Iaboni et al., 1995; Milich et al., 1994). Although Milich et al. (1994) and Hartung et al. (2002) found significant GNG commission errors for ADHD/C groups, these effects were marginal and held only for adolescent boys. A study by Iaboni et al. (1995) involving children aged 8 to 13 also found positive relations between ADHD/C and GNG errors of commission, although the task was modified from Newman and Kosson's (1996) task in important ways for this age group. Specifically, Iaboni and colleagues (1995) used fewer stimuli numbers (6 vs. 8), had stimuli numbers restricted to a lower range (1 to 40 vs. 14-96), left stimuli 
on the screen for longer periods (4 seconds vs. 3), and continued for more trials (12 vs. 10). In the current study, no such modifications were made, making this the only study to use Newman and Kosson's (1996) original task criteria with this ADHD age group. It is therefore possible that although the cognitive requirements of this task were appropriate for adolescents, they were too demanding for this age group, thus attenuating the potential impact motivational contingencies had on inhibitory performance.

However, despite the limited ability of these tasks to replicate inhibitory deficit findings in $\mathrm{ADHD} / \mathrm{C}$ children, the contrast between task requirements makes findings regarding the ADHD/I of importance, given that similar deficits were found across these tasks. Potentially important differences across tasks did little to change ADHD/I responding, and further supports a pervasive, underlying processing deficit unique to this subtype.

\section{Implications}

Among the primary implications from this body of work is the need to reconsider descriptions of the diagnostic classification of ADHD/I. The current conceptualization of ADHD endorsed by the DSM-IV implies that our understanding of the ADHD/C and ADHD/I subtypes is advanced by considering them as being phenotypically similar. However, findings from the present study and others (e.g., O’Driscoll et al., 2005) strongly suggest dramatic behavioral differences between the two subtypes, including response speed, sensitivity to environmental cues, and level of behavioral inhibition. Such basic phenotypic differences suggest that these groups may have differential underlying pathologies.

This line of research raises important questions regarding the current diagnostic system in which ADHD/C and ADHD/I are assumed to be subtypes of the same disorder. The goal of all classifications systems is to advance our understanding of the disorders identified. In terms of the 
$\mathrm{ADHD} / \mathrm{I}$ group, the question becomes whether our understanding of this group is enhanced further by considering it as similar to, or distinct from, the ADHD/C group. For example, a major purpose of a classification system is to synthesize and reflect the best available information with the goal of promoting the most effective treatment. In the present study, the slow, cautious, and ineffective responses observed among children with ADHD/I suggest that the traditional behavioral treatment for children with $\mathrm{ADHD} / \mathrm{C}$, with a reliance on response-cost procedures to decrease disruptive and disinhibited behavior, may be less effective in treating the primary symptoms of children with ADHD/I as they are unlikely to exhibit these types of behaviors (see, for example, Pfiffner, Mikami, Huang-Pollock, Easterlin, Zalecki, \& McBurnett, 2007). Further research is clearly needed to determine whether interventions designed to directly address the inattentive and sluggish performance of those with ADHD/I would be more efficacious than the treatments traditionally employed for children with ADHD/C.

\section{Limitations and Future Directions}

The results of the current study support the notion that ADHD/I and ADHD/C may have important divergence with regard to presentation of symptoms. It is important for future investigations to continue this line of research, identifying the specific pathological mechanisms underlying the observable symptoms, be they deficient motivation, increased anxiety, or discrete neurological systems. For instance, use of explicit measures of sluggish cognitive tempo in conjunction with behavioral inhibition tasks would allow for direct examination of the contribution processing deficits make to task performance. It may well be that increased reaction times and inhibited responding are better accounted for by the inability of ADHD/I children to adequately integrate information. Moreover, although the CBCL parent reports available in the current study were sufficient to rule out the possibility that anxiety was accounting for group 
differences, it is recognized that use of a parent-report anxiety measure may not be the optimal method for assessing internalizing pathology in child populations (Rey, Schrader, \& MorrisYates, 1992). Use of self-report anxiety measures in conjunction with parent report would eliminate the possibility that anxiety contributes to ADHD subtype differences in performance.

Due to the failure to find behavioral inhibition deficits in the ADHD/C group on the CRT and GNG tasks, the field would also benefit from comparison of the current tasks with other commonly used behavioral inhibition tasks in the ADHD literature, such as the stop task, to identify potential divergence in the underlying constructs these tasks measure. Additionally, beyond continued exploration of the ADHD subtype differences using behavioral inhibition and motivational tasks, use of sophisticated neurocognitive test batteries and neuroimaging studies will help explore other potential areas of divergence. Recent work has begun to test hypothesized links between neural systems and symptoms (Solanto et al., 2007), although few neurocognitive tests have been utilized to test subtype differences. It is possible that through further exploration, other important differences between the ADHD subtypes will continue to emerge. Finally, it is recognized that research has indicated that gender may impact ADHD subtype differences in inhibitory control (Nigg et al., 2002). While the current study lacked sufficient statistical power to conduct such analyses, this remains an important issue worthy of exploration.

In summary, the current results indicate that important behavioral differences exist between the ADHD/C and ADHD/I subtypes, and suggest that future research continue to explore whether distinct diagnostic categories may better capture the nature of these two disorders. Such a perspective may generate questions and treatment approaches that are not now being considered because the two groups are conceptualized as subtypes of the same disorder. 


\section{References}

Abroms, B. D., Fillmore, M. T., \& Marczinski, C. A. (2003). Alcohol-induced impairment of behavioral control: Effects on the alteration and suppression of prepotent responses. Journal of Studies on Alcohol, 64, 687-695.

Achenbach, T. M. (1991). Manual for the Child Behavior Checklist/4-18 and 1991 profile. Burlington, University of Vermont Department of Psychiatry.

American Psychiatric Association (1994). Diagnostic and statistical manual of mental disorders (DSM-IV) (4th ed.). Washington, DC: American Psychiatric Association.

Baeyens, D., Roeyers, H., \& Vande Walle, J. (2006). Subtypes of AttentionDeficit/Hyperactivity Disorder (ADHD): Distinct or Related Disorders Across Measurement Levels? Child Psychiatry and Human Development, 36, 403-417.

Barkley, R. A. (1997). Behavioral inhibition, sustained attention, and executive functions: Constructing a unifying theory of ADHD. Psychological Bulletin, 121, 65-94.

Barkley, R. A. (2001). The inattentive type of ADHD as a distinct disorder: What remains to be done. Clinical Psychology: Science and Practice, 8, 489-493.

Barkley, R. A. (2006). Attention-deficit hyperactivity disorder, 3rd ed.: A handbook for diagnosis and treatment. New York, NY: Guilford Press.

Barr, C. L., Wigg, K. G., Bloom, S., Schachar, R., Tannock, R., Roberts, W., Malone, M., \& Kennedy, J. L. (2000). Further evidence from haplotype analysis for linkage of the dopamine D4 receptor gene and ADHD. American Journal of Medical Genetics, 96, 262267.

Bauermeister, J. J., Barkley, R. A., \& Martinez, J. V. (2005). Time estimation and performance on reproduction tasks in subtypes of children with attention deficit hyperactivity disorder. 
Journal of Clinical Child and Adolescent Psychology, 34, 151-162.

Carlson, C. L., \& Mann, M. (2000). Attention-deficit/hyperactivity disorder, predominately inattentive subtype. Child and Adolescent Psychiatric Clinics of North America, 9, 499510.

Carlson, C. L., \& Mann, M. (2002). Sluggish cognitive tempo predicts a different pattern of impairment in the attention deficit hyperactivity disorder, primarily inattentive type. Journal of Clinical Child and Adolescent Psychology, 31, 123-129.

Carr, L. A., Nigg, J. T., \& Henderson, J. M. (2006). Attentional versus motor inhibition in adults with attention deficit/hyperactivity disorder. Neuropsychology, 4, 430-441.

Castellanos, F. X., Sonuga-Barke, E. J. S., Milham, M. P., \& Tannock, R. (2006). Characterizing cognition in ADHD: Beyond executive dysfunction. Trends in Cognitive Sciences, $10,117-123$.

Castellanos, F. X., \& Tannock, R. (2002). Neuroscience of attention-deficit/hyperactivity disorder: the search for endophenotypes. Nature Reviews: Neuroscience, 3, 617-628.

Conners, C. K. (1997). Conners' Rating Scales—Revised: Technical Manual. North Tonawanda, NY: Multi-Health Systems.

Daly, G., Hawi, Z., Fitzgerald, M., \& Gill, M. (1999). Mapping susceptibility loci in attention deficit hyperactivity disorder: Preferential transmission of parental alleles at DAT1, DBH and DRD5 to affected children. Molecular Psychiatry, 4, 192-196.

Diamond, A. (2005). Attention-deficit disorder (attention-deficit/hyperactivity disorder without hyperactivity): A neurobiologically and behaviorally distinct disorder from attention deficit/hyperactivity (with hyperactivity). Development and Psychopathology, 17, 807825. 
Durston, S., Davidson, M. C., Mulder, M. J., Spicer, J. A., Galvan, A., Tottenham, N., Scheres, A., Castellanos, F. X., vanEngeland, H., \& Casey, B. J. (2007). Neural and behavioral correlates of expectancy violations in attention-deficit hyperactivity disorder. Journal of Child Psychology and Psychiatry, 48, 881-889.

Epstein, J. N., Johnson, D. E., Varia, I. M., \& Conners, C. K. (2001). Neuropsychological assessment of response inhibition in adults with ADHD. Journal of Clinical and Experimental Neuropsychology, 23, 362-371.

Faraone, S. V., Biederman, J., Weber, W., \& Russell, R. L. (1998). Psychiatric, neuropsychological, and psychosocial features of DSM-IV subtypes of attentiondeficit/hyperactivity disorder: Results from a clinically referred sample. Journal of the American Academy of Child and Adolescent Psychiatry, 37, 185-193.

Fillmore, M. T., Marczinski, C. A., \& Bowman, A. M. (2005). Acute tolerance to alcohol effects on inhibitory and activational mechanisms of behavioral control. Journal of Studies on Alcohol, 66, 663-672.

Flory, K., Milich, R., Lorch, E. P., Hayden, A. N., Strange, C., \& Welsh, R. (2006). Online story comprehension among children with ADHD: Which core deficits are involved? Journal of Abnormal Child Psychology, 34, 853-865.

Gray, J. A. (1991). The neuropsychology of temperament. In J. Strelau and A. Angleitner (Eds.), Explorations in temperament: International perspectives on theory and measurement (pp. 105-128). Plenum Press: New York, NY.

Hartman, C.A., Willcutt, E.G., Rhee, S.H., \& Pennington, B.F. (2004). The relation between sluggish cognitive tempo and DSM-IV ADHD. Journal of Abnormal Child Psychology, 32, 491-503. 
Hartung, C. M., Milich, R., Lynam, D. R., \& Martin, C. A. (2002). Understanding the relations among gender, disinhibition, and disruptive behavior in adolescents. Journal of Abnormal Psychology, 111, 659-664.

Hinshaw, S. P. (2001). Is the inattentive type of ADHD a separate disorder? Clinical Psychology: Science and Practice, 8, 498-501.

Hinshaw, S. P. (2002). Preadolescent girls with attention-deficit/hyperactivity disorder: I. Background characteristics, comorbidity, cognitive and social functioning, and parenting practices. Journal of Consulting and Clinical Psychology, 70, 1086-1098.

Huang-Pollock, C. L., Mikami, A. Y., Pfiffner, L., \& McBurnett, K. (2007). ADHD subtype differences in motivational responsivity but not inhibitory control: Evidence from a reward-based variation of the stop signal paradigm. Journal of Clinical Child and Adolescent Psychology, 36, 127-136.

Iaboni, F., Douglas, V. I., \& Baker, A. G. (1995). Effects of reward and response costs on inhibition in ADHD children. Journal of Abnormal Psychology, 104, 232-240.

Iaboni, F., Douglas, V. I., \& Ditto, B. (1997). Psychophysiological response of ADHD children to reward and extinction. Psychophysiology, 34, 116-123.

Lahey, B. B. (2001). Should the combined and predominantly inattentive types of ADHD be considered distinct and unrelated disorders? Not now, at least. Clinical Psychology: Science and Practice, 8, 494-497.

Lahey, B. B., Pelham, W. E., Loney, J., Lee, S. S., \& Willcutt, E. (2005). Instability of the DSMIV Subtypes of ADHD From Preschool Through Elementary School. Archives of General Psychiatry, 62, 896-902.

Lijffijt, M., Kenemans, J. L., Verbaten, M. N., \& van Engeland, H. (2005). A Meta-Analytic 
Review of Stopping Performance in Attention-Deficit/Hyperactivity Disorder: Deficient Inhibitory Motor Control? Journal of Abnormal Psychology, 114, 216-222.

Logan, G. D. (1994). On the ability to inhibit thought and action: A users' guide to the stop signal paradigm. In D. Dagenbach \& T. H. Carr (Eds.), Inhibitory processes in attention, memory, and language (pp. 189-239). Academic Press, Toronto. 189-239

Logan, G. D., \& Cowan, W. B. (1984). On the ability to inhibit thought and action: A theory of an act of control. Psychological Review, 91, 295-327.

Logan, G. D., \& Irwin, D. E. (2000). Don’t look! Don't touch! Inhibitory control of eye and hand movements. Psychonomic Bulletin \& Review, 7, 107-112.

Lorch, E. P., Sanchez, R. P., van den Broek, P., Milich, R., Murphy, E. L., Lorch, R. F., Welsh, R. (1999). The relation of story structure properties to recall of television stories in young children with attention-deficit hyperactivity disorder and nonreferred peers. Journal of Abnormal Child Psychology, 27, 293-309.

Luman, M., Oosterlaan, J. A., \& Sergeant, J. (2005). The impact of reinforcement contingencies on AD/HD: A review and theoretical appraisal. Clinical Psychology Review, 25, 183-213.

MacCoon, D. G., Wallace, J. F., \& Newman, J. P. (2004). Self-regulation: Context-appropriate balanced attention. In K. D. Vohs and R. F. Baumeister (Eds.), Handbook of selfregulation: Research, theory, and applications (pp. 422-444). New York, NY: Guilford Press.

Maedgen, J., \& Carlson, C. L. (2000). Social functioning and emotional regulation in the attention deficit hyperactivity disorder subtypes. Journal of Clinical Child Psychology, 29, 30-42.

Marczinski, C. A., \& Fillmore, M. T. (2003). Preresponse cues reduce the impairing effects of 
alcohol on the execution and suppression of responses. Experimental and Clinical Psychopharmacology, 11, 110-117.

Marshall, R. M., Hynd, G. W., Handwerk, M. J., \& Hall, J. (1997) Academic underachievement in ADHD subtypes. Journal of Learning Disabilities, 30, 635-642.

McBurnett, K., Pfiffner, L. J., Willcutt, E., Tamm, L., Lerner, M., Ottolini, Y. L., et al. (1999). Experimental cross-validation of $D S M-I V$ types of attention-deficit/hyperactivity disorder. Journal of the American Academy of Child and Adolescent Psychiatry, 38, 1724.

Milich, R., Balentine, A. C., Lynam, D. R. (2001). ADHD combined type and ADHD predominantly inattentive type are distinct and unrelated disorders. Clinical Psychology: Science and Practice, 8, 463-488.

Milich, R., Hartung, C. M., Martin, C. A., \& Haigler, E. D. (1994). Behavioral disinhibition and underlying processes in adolescents with disruptive behavior disorders. In D. K. Routh (Ed.), Disruptive behavior disorders in childhood (pp. 109-138). New York: Plenium.

Newman, J. P., \& Kosson, D. (1996). Passive avoidance learning in psychopathic and nonpsychopathic children. Journal of Abnormal Psychology, 95, 2257-2263.

Newman, J. P., \& Wallace, J. F. (1993). Diverse pathways to deficient self-regulation: Implications for disinhibitory psychopathology in children. Clinical Psychology Review, 13, 699-720.

Nichols, S. L., \& Waschbusch, D. A. (2004). A review of the validity of laboratory cognitive tasks used to assess symptoms of ADHD. Child Psychiatry and Human Development, 34, 297-315.

Nigg, J. T. (2000). On inhibition/disinhibition in developmental psychopathology: Views from 
cognitive and personality psychology and a working inhibition taxonomy. Psychological Bulletin, 126, 200-246.

Nigg, J. T. (2001). Is ADHD an inhibitory disorder? Psychological Bulletin, 127, 571-598.

Nigg, J. T., Blaskey, L. G., Huang-Pollock, C. L., \& Rappley, M. D. (2002). Neuropsychological executive functions and DSM-IV ADHD subtypes. Journal of the American Academy of Child \& Adolescent Psychiatry, 41, 59-66.

Nigg, J. T. (2006). What causes ADHD?: Understanding what goes wrong and why. New York, NY: Guilford Press.

O'Driscoll, D. A., Depatie, L., Holahan, A. V., Savion-Lemieux, T., Barr, R. G., Jolicoeur, C., \& Douglas, V. I. (2005). Executive functions and methylphenidate response in subtypes of Attention-deficit/hyperactivity disorder. Biological Psychiatry, 57, 1452-1460.

Oosterlaan, J., Logan, G. D., \& Sergeant, J. A. (1998). Response inhibition in AD/HD, CD, comorbid $\mathrm{AD} / \mathrm{HD}+\mathrm{CD}$, anxious and control children: a meta-analysis of studies with the stop task. Journal of Child Psychology and Psychiatry, 39, 411-425.

Patterson, C., \& Newman, J. P. (1993). Reflectivity and learning from aversive events: Toward a psychological mechanism for the syndromes of disinhibition. Psychological Review, 100, 716-736.

Pelham W. E. (2001). Are ADHD/I and ADHD/C the same or different? Does it matter? Clinical Psychology: Science and Practice, 8, 502-506.

Pennington, B. E, \& Ozonoff, S. (1996). Executive functions and developmental psychopathology. Journal of Child Psychology and Psychiatry, 37, 51-87.

Pliszka, S. R., Bocherding, S. H., Spratley, K., Leon, S., \& Irick, S. (1997). Measuring inhibitory control in children. Journal of Developmental and Behavioral Pediatrics, 18, 254-259. 
Pliszka, S. R., Hatch, J. P., Bocherding, S. H., \& Rogeness, G. A. (1993). Classical conditioning in children with attention deficit hyperactivity disorder (ADHD) and anxiety disorders: A test of Quay's model. Journal of Abnormal Child Psychology, 21, 411-523.

Pfiffner, L. J., Mikami, A. Y., Huang-Pollock, C. L., Easterlin, B., Zalecki, C., \& McBurnett, K. (2007). A randomized, controlled trial of integrated home-school behavioral treatment for ADHD, predominantly inattentive type. Journal of the American Academy of Child and Adolescent Psychiatry, 46, 1041-1050.

Quay, H. (1988). The behavioral reward and inhibition system in childhood behavior disorder. Elmsford, NY: Pergamon Press.

Quay, H. C. (1997). Inhibition and attention deficit hyperactivity disorder. Journal of Abnormal Child Psychology, 25, 7-13.

Rey, J.M., Schrader, E. \& Morris-Yates, A., (1992). Parent-child agreement on children's behaviours reported by the Child Behavior Checklist (CBCL). Journal of Adolescence $15,219-230$.

Riccio, C. A., Homack, S., Jarratt-Pizzitola, K., \& Wolfe, M. E. (2006). Differences in academic and executive function domains among children with ADHD Predominantly Inattentive and Combined Types. Archives of Clinical Neuropsychology, 21, 657-667.

Rommelse, N. N. J., Altink, M. E., Sonneville, L. M. J., Buschgens, C. J. M., Buitelaar, J., Oosterlaan, J., \& Sergeant, J. A. (2007). Are motor inhibition and cognitive flexibility dead ends in ADHD? Journal of Abnormal Child Psychology, 35, 957-967.

Rowe, D. C., Stever, C., \& Gard, J. M. C. (1998). The relation of the dopamine transporter gene (DAT1) to symptoms of internalizing disorders in children. Behavior Genetics, 28, 215225. 
Smoller, J. W., Biederman, J., Arbeitman, L., Doyle, A. E., Fagerness, J., Perlis, R. H., Sklar, P., Faraone, S. V. (2006). Association between the 5HT1B receptor gene (HTR1B) and the inattentive subtype of ADHD. Biological Psychiatry, 59, 460-467.

Solanto, M. V., Gilbert, S. N., Raj, A., Zhu, J., Pope-Boyd, S., Stepak, B., Vail, L., \& Newcorn, J. H. (2007). Neurocognitive functioning in AD/HD, predominantly inattentive and combined subtypes. Journal of Abnormal Child Psychology, 35, 729-744.

Weiss, M. D., Worling, D. E., \& Wasdell, M. B. (2003). A chart review study of the Inattentive and Combined Types of ADHD. Journal of Attention Disorders, 7, 1-9.

Weller, E. B., Weller, R. A., Rooney, M. T., \& Fristad, M. A. (1999). ChIPS. Washington, DC: American Psychiatric Association.

Wilens, T., Pelham, W., Stein, M., Conners, C., Abikoff, H., Atkins, M., et al. (2003, April). ADHD treatment with once-daily OROS methylphenidate: Interim 12-month results from a long-term open-label study. Journal of the American Academy of Child \& Adolescent Psychiatry, 42, 424-433. 
Table 1

Diagnostic information by group

\begin{tabular}{|c|c|c|c|c|c|}
\hline & $\begin{array}{l}\text { Comparison } \\
\qquad(n=21)\end{array}$ & $\begin{array}{l}\text { ADHD/I } \\
(n=17)\end{array}$ & $\begin{array}{l}\text { ADHD/C } \\
(n=37)\end{array}$ & & \\
\hline$\underline{\text { Variable }}$ & Mean $(\mathrm{SD})$ & Mean (SD) & Mean (SD) & $\mathrm{F}$ & t result \\
\hline Age diagnosed with ADHD (years) & & $8.2(0.9)$ & $7.1(2.2)$ & 3.08 & \\
\hline DSM-IV attention & $.52(1.1)$ & $6.2(2.6)$ & $7.1(2.0)$ & $80.48 * * *$ & $\mathrm{a}$ \\
\hline DSM-IV hyperactivity & $.38(0.7)$ & $1.5(1.5)$ & $5.4(2.3)$ & $60.41 * * *$ & $\mathrm{~b}$ \\
\hline DSM-IV oppositional/defiant & $.19(0.4)$ & $1.1(1.3)$ & $3.4(2.2)$ & $28.01 * * *$ & $\mathrm{~b}$ \\
\hline Connors oppositionality & $42.8(3.9)$ & $50.4(8.3)$ & $61.4(12.4)$ & $25.40 * * *$ & $b$ \\
\hline Connors cognitive problems/inattention & $45.1(3.8)$ & $71.5(9.3)$ & $73.0(9.1)$ & $88.66 * * *$ & a \\
\hline Connors hyperactivity & $47.8(5.1)$ & $53.1(11.6)$ & $76.5(10.2)$ & $74.65 * * *$ & $\mathrm{~b}$ \\
\hline Connors ADHD total & $44.7(3.3)$ & $68.5(8.5)$ & $74.7(6.8)$ & $145.44 * * *$ & $\mathrm{c}$ \\
\hline CBCL aggression & $51.1(3.9)$ & $54.4(5.9)$ & $62.2(10.0)$ & $15.08 * * *$ & $\mathrm{~b}$ \\
\hline CBCL delinquency & $51.4(2.8)$ & $53.2(5.7)$ & $59.0(8.8)$ & $9.20 * * *$ & $\mathrm{~b}$ \\
\hline CBCL anxiety & $52.3(4.49)$ & $56.9(20.1)$ & $60.0(8.5)$ & 3.05 & \\
\hline
\end{tabular}

Note. $* p<.05, * * p<.01, * * * p<.001$. ADHD/I = ADHD inattentive. ADHD/C $=$ ADHD combined. DSM scores based on semi- 
structured interview. a. Comparison significantly different from all other groups. b. ADHD/C significantly different from all other groups. c. Comparison significantly different from ADHD/I, and ADHD/I significantly different from ADHD/C. 
Table 2

Demographic information by group

\begin{tabular}{|c|c|c|c|c|c|c|}
\hline & $\begin{array}{l}\text { Comparison } \\
\qquad(n=21)\end{array}$ & $\begin{array}{l}\text { ADHD/I } \\
(n=17)\end{array}$ & $\begin{array}{l}\mathrm{ADHD} / \mathrm{C} \\
(n=37)\end{array}$ & & & \\
\hline$\underline{\text { Variable }}$ & Mean (SD) & Mean (SD) & Mean (SD) & $\mathrm{F}$ & $X^{2}$ & $p$ \\
\hline Age (months) & $131.2(11.3)$ & $125.1(13.6)$ & $132.2(13.5)$ & 1.79 & & .174 \\
\hline Gender ( $\%$ male $)$ & 66.7 & 64.7 & 83.8 & & 3.23 & .199 \\
\hline Race (\% white) & 85.7 & 76.5 & 89.2 & & 1.51 & .470 \\
\hline Grade level & $5.5(1.1)$ & $4.7(1.2)$ & $5.2(1.2)$ & 2.15 & & .124 \\
\hline Mother's education & $16.4(1.7)$ & $15.3(2.0)$ & $15.7(2.0)$ & 1.10 & & .338 \\
\hline Father's education & $16.0(2.0)$ & $16.0(3.3)$ & $14.6(3.6)$ & 1.95 & & .150 \\
\hline KBIT vocabulary & $107.2(11.8)$ & $102.9(13.4)$ & $101.2(10.9)$ & 1.77 & & .178 \\
\hline KBIT matrices & $120.1(13.3)$ & $109.7(12.4)$ & $108.2(14.8)$ & 5.15 & & .008 \\
\hline
\end{tabular}

Note. $\mathrm{ADHD} / \mathrm{I}=\mathrm{ADHD}$ inattentive. $\mathrm{ADHD} / \mathrm{C}=\mathrm{ADHD}$ combined. $\mathrm{KBIT}=$ Kaufman Brief Intelligence Test. Parental education is provided in years. KBIT matrices, both ADHD subgroups obtained lower scores than healthy comparison children. No differences were observed among ADHD subgroups, however. 
Table 3

CRT task performance and reaction time data across comparison and ADHD subtype groups

\begin{tabular}{|c|c|c|c|c|c|}
\hline & $\begin{array}{l}\text { Comparison } \\
\qquad(n=21)\end{array}$ & $\begin{array}{l}\text { ADHD/I } \\
\quad(n=17)\end{array}$ & $\begin{array}{l}\text { ADHD/C } \\
(n=37)\end{array}$ & & \\
\hline$\underline{\text { Variable }}$ & Mean (SD) & Mean (SD) & Mean (SD) & $\mathrm{F}$ & $\mathrm{t}$ result \\
\hline CRT proportion IF GO & $.12(.13)$ & $.05(.09)$ & $.13(.17)$ & 1.91 & \\
\hline CRT proportion IF NOGO & $.07(.11)$ & $.04(.04)$ & $.07(.12)$ & .58 & \\
\hline CRT cue dependency & $.05(.08)$ & $.01(.07)$ & $.06(.10)$ & 1.82 & \\
\hline CRT reaction time GO & $350.95(43.80)$ & $418.59(58.46)$ & $379.28(66.35)$ & $6.16^{* *}$ & $\mathrm{a}$ \\
\hline CRT reaction time NOGO & $370.15(53.51)$ & $443.48(66.96)$ & $409.28(83.09)$ & $4.88^{*}$ & $\mathrm{a}$ \\
\hline CRT reaction time SD GO & $89.90(24.02)$ & $106.04(34.89)$ & $106.11(36.12)$ & 1.83 & \\
\hline CRT reaction time SD NOGO & $84.85(35.30)$ & $107.81(36.34)$ & $106.91(36.12)$ & $2.77 *$ & $\mathrm{~b}$ \\
\hline
\end{tabular}

Note. ${ }^{*} p<.05, * * p<.01, * * * p<.001$. CRT proportion IF GO = Cued reaction time task proportion of inhibitory failures following go cues. CRT proportion IF NOGO = Cued reaction time task proportion of inhibitory failures following no-go cues. CRT cue dependency $=$ Difference between proportion of inhibitory failures following go and no-go cues. CRT reaction time GO = Reaction times to go targets following go cue. CRT reaction time $\mathrm{NOGO}=$ Reaction times to go targets following no-go cue. CRT reaction time $\mathrm{SD}$ GO = Reaction time variability following go cue. CRT reaction time SD NOGO = Reaction time variability following no-go cue 
a. ADHD/I significantly different from Comparison. b. Comparison significantly different from all other groups. 
Table 4

GNG task performance and reaction time data across comparison and ADHD subtype groups

\begin{tabular}{|c|c|c|c|c|c|}
\hline & $\begin{array}{l}\text { Comparison } \\
\qquad(n=21)\end{array}$ & $\begin{array}{l}\text { ADHD/I } \\
(n=17)\end{array}$ & $\begin{array}{l}\text { ADHD/C } \\
(n=37)\end{array}$ & & \\
\hline$\underline{\text { Variable }}$ & Mean (SD) & Mean (SD) & Mean (SD) & $\mathrm{F}$ & $\mathrm{t}$ result \\
\hline GNG omission errors & $5.00(4.30)$ & $8.59(5.29)$ & $4.08(4.31)$ & $5.81 * *$ & a \\
\hline GNG commission errors & $17.10(7.89)$ & $17.06(7.78)$ & $19.68(9.34)$ & .85 & \\
\hline GNG reaction time & $944.70(199.24)$ & $1123.08(190.85)$ & $953.64(167.36)$ & $5.93 * *$ & $\mathrm{a}$ \\
\hline GNG reaction time SD & $434.00(102.20)$ & $539.51(93.41)$ & $484.05(119.69)$ & $4.37 * *$ & $\mathrm{~b}$ \\
\hline
\end{tabular}

Note. ${ }^{*} p<.05, * * p<.01, * * * p<.001$. GNG $=$ Go/no-go task. a. ADHD/I significantly different than all other groups $(p<.05)$. b. ADHD/I significantly different from Comparison $(p<.05)$ and marginally significantly different from ADHD/C $(p<.06)$ groups. 


\section{Figure Caption}

Figure 1. CRT cue-dependency vs. CRT reaction time differences between comparison and ADHD subtypes. CRT PIF GO = Cued reaction time task proportion of inhibitory failures following go cues. CRT PIF NOGO = Cued reaction time task proportion of inhibitory failures following no-go cues. CRT RT GO = Cued reaction time task mean reaction time to go targets following go cues. CRT RT NOGO = Cued reaction time task mean reaction time to go targets following no-go cues. 

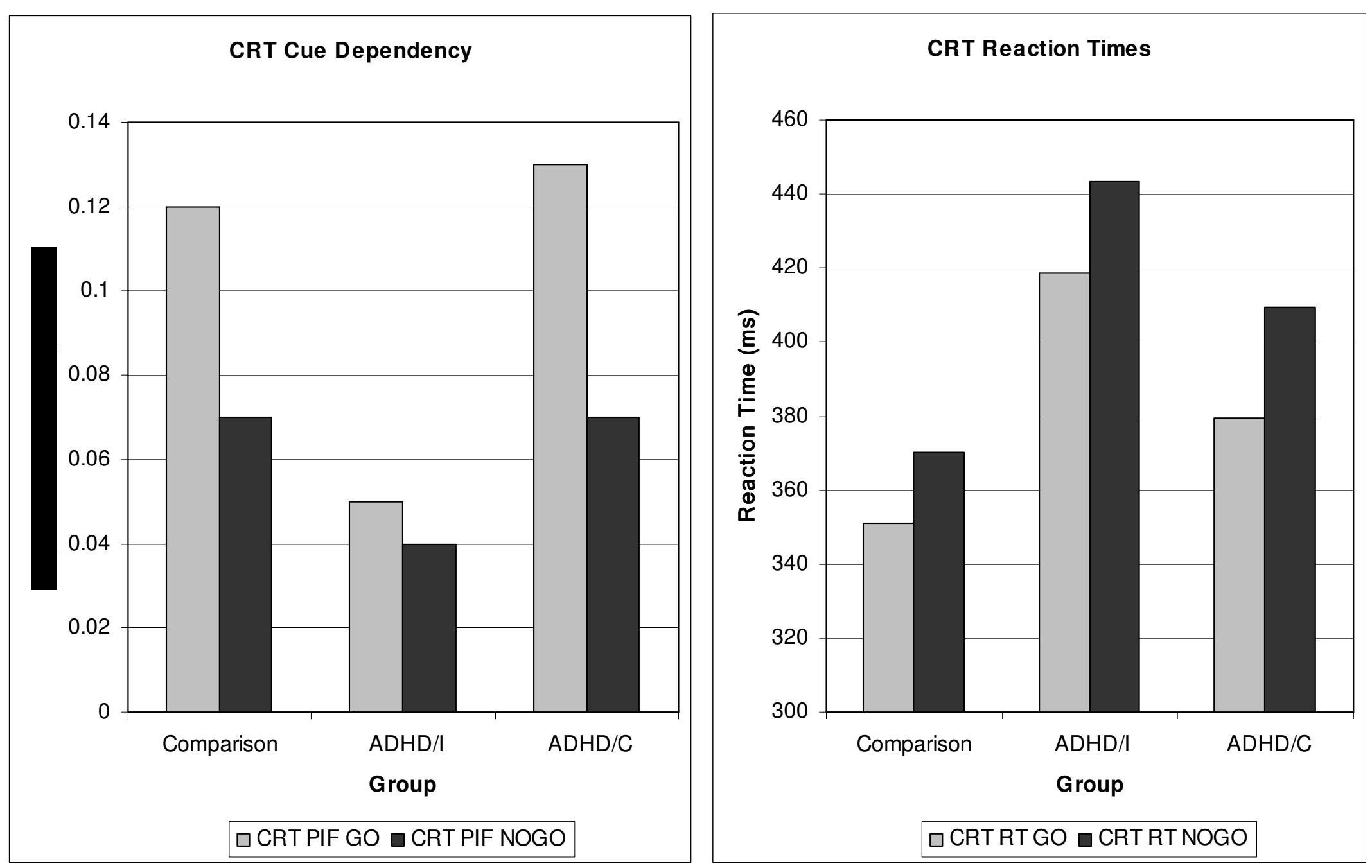